\title{
sciendo
}

\section{A Video-Based Method to Quantify Stroke Synchronisation in Crew Boat Sprint Kayaking}

\author{
by \\ Cheryl Sihui Tay', Pui Wah Kong ${ }^{1}$
}

\begin{abstract}
The study aimed to quantify stroke synchronisation in two-seater crew boat sprint kayaking (K2) using a video-based method, and to assess the intra- and inter-rater reliabilities of this method. Twelve sub-elite sprint kayakers (six males and six females) from a national team were paired into six single-gender K2 crews. The crews were recorded at $120 \mathrm{~Hz}$ with a sagittal-view video camera during 200-m time trials. Video analysis identified four meaningful positions of a stroke (catch, immersion, extraction and release). The timing difference (termed "offset") between the front and back paddlers, within each K2, at each stroke position was calculated, with zero offset indicating perfect synchronisation. Results showed almost perfect intra-rater reliability of this method. The intra-class correlation (ICC) ranged from .87 to 1.00, and standard error of measurement (SEM) from 0 to 5 milliseconds (ms). Inter-rater reliability was substantial to almost perfect (ICC $.72-.94$, SEM $2-6 \mathrm{~ms}$ ). On average, 35 strokes were analysed for each crew and the mean offset was $17 \mathrm{~ms}$, or $5.7 \%$ of water phase duration. Crews were more synchronised at the catch (11 $\mathrm{ms}$, $3.8 \%$ ) than the release (21 ms, 7.2\%). However, the stroke synchronisation profiles of the six sub-elite crews varied considerably from each other. For example, the best performing male and female crews had directly contrasting profiles. This suggests that there is no universal stroke synchronisation profile for well-trained sprint kayakers. This video-based method may aid future investigations on improving performance.
\end{abstract}

Key words: paddling, team-boat, technique, performance, reliability.

\section{Introduction}

Crew boat racing has a long history in many parts of the world through various forms such as kayaking, rowing, outrigger canoeing and dragon boat racing. In a crew boat, two or more individuals use oars or paddles to propel the boat. Sprint kayaking is an Olympic sport where the two-seater (K2) and four-seater (K4) crew boats race, in addition to the one-seater kayak (K1). Sprint kayakers perform forward strokes cyclically on alternate sides with a double-bladed paddle. There are complexities associated with racing in crew boats that are not found in the single boat, such as the need for paddling stroke synchronisation.

To an observer, a crew is synchronised when each member strokes in time from the catch to exit, which are positions that define the water

phase of a stroke cycle (Szanto, 2010). Due to the design of sprint kayak crew boats where seats are in close proximity, crews must have some degree of stroke synchronisation to avoid clashing their paddles. In addition, it is commonly believed that synchronised strokes are important for crew boat racing performance (King and de Rond, 2011). However, photographic evidence shows that some of the best sprint kayak crews display slight asynchronicity (out-of-sync strokes), whereby the paddler(s) seated at the back start the stroke earlier and finish later than the front paddler (Tellez et al., 2015). It has been theorised that stroke asynchronicity could be beneficial for racing performance, as power lost to boat velocity fluctuations in the forward direction are reduced (Martin and Bernfield, 1980). Yet, the only

\footnotetext{
1 - Physical Education and Sports Science Academic Group, National Institute of Education, Nanyang Technological University, Singapore.
} 
supporting experimental data were collected only more recently from coupled rowing ergometers on land (de Brouwer et al., 2013). Aside from movement differences between rowing and kayaking, ergometry removes the need to balance in the narrow, streamlined racing boats, and hence cannot be applied directly to on-water sprint kayaking. It is thus important to develop methods that are practical to analyse on-water performance during which crews must maintain balance in streamlined racing boats as narrow as $0.37 \mathrm{~m}$.

The research on crew boat sprint kayaking is lacking as the focus has traditionally been on K1 (Jackson, 1995; Michael et al., 2009). There have only been two peer-reviewed studies on crew boat sprint kayaking. One study compared three $\mathrm{K} 4$ combinations from a national team during 500-m time trials (Robinson et al., 2011). One particular combination was faster (4.9 $\mathrm{s}, \quad 4.6 \%$ ) and had higher five-stroke peak acceleration $\left(5.5 \mathrm{~m} / \mathrm{s}^{2}\right.$ versus $\left.3.5 \mathrm{~m} / \mathrm{s}^{2}\right)$ than the other two crews who tied at $1 \min 46.8 \mathrm{~s}$. Although the fastest crew combination was described as more synchronised than the other two crews, no further details were provided. Another study found that crew boats had more even $250-\mathrm{m}$ split timings than $\mathrm{K} 1 \mathrm{~s}$ at the 2004 to 2011 editions of the World Championships (Borges et al., 2009). No published studies have specifically investigated stroke synchronisation in crew boat sprint kayaking.

Therefore, the aims of this study were to 1) quantify stroke synchronisation in crew boat sprint kayaking using a video-based method, 2) assess the intra- and inter-rater reliabilities of this method, and 3) identify stroke synchronisation patterns of sprint kayak K2 crews from a national team. Although video-based methods might seem primitive as compared to more advanced methods such as paddle instrumentation, they are relatively accessible, inexpensive, and userfriendly. It was hypothesised that the proposed video-based method would be reliable in quantifying stroke synchronisation of sub-elite sprint kayak K2 crews.

\section{Methods}

\section{Participants}

Twelve sprint kayakers (6 males and 6 females) from a national team participated in this study. The participants competed internationally and trained for 25 to 30 hours a week. Table 1 lists the participant characteristics and their individual K1 200-m performance. All procedures were in accordance with the Declaration of Helsinki regarding research involving human participants. This study received ethical approval from the Nanyang Technological University Institutional Review Board (IRB-2014-05-027). All participants gave their written consent.

\section{Design and Procedures}

This study utilised an observational design and did not involve manipulations or deliberate instructions to the participants. Participants were paired into six single-gender K2 crews by their coach in preparation for selections towards a regional competition. The study was conducted at a reservoir training base with a 1000$\mathrm{m}$ race course. Each crew was assigned a K2 sprint kayak of similar make, and weighed to ensure it met the minimum competition mass of $18.00 \mathrm{~kg}$. Where necessary, additional masses were secured. The seat and footrest fittings were customised by each participant. Participants used their personal paddles. Prior to the 200-m time trial, participants performed their self-selected warm-up on land, followed by on water in their respective $\mathrm{K} 2$ crews.

Performance of the 200-m time trial was recorded at $120 \mathrm{~Hz}$ from the right-hand side sagittal view using a high-speed digital video camera (Casio EX-FH 100, Casio, Shibuya, Tokyo, Japan). The camera was operated by a researcher on a power boat accompanying alongside the $\mathrm{K} 2$ crew from about 9-m away. Sagittal view recordings show the paddle blade positions of both K2 athletes concurrently, unlike other views (e.g. frontal or dorsal) where the paddle blades may be obstructed. This method had been used to study elite K4 athletes (Robinson et al., 2011).

Photo 1 shows an annotated screen capture from the video. The capture space was about $8.5 \mathrm{~m}$ wide, and encompassed the tip of the boat (bow), the approaching buoy markers, and the release position of the back paddler's blade. Performance time was measured from the first instance of forward movement of the bow, until the bow reached the pair of finishing buoy markers. This method of obtaining performance time eliminates the reaction time errors of the participants to the "Go" command. Based on data 
from ten pilot trials, standard error of measurement for performance time was $0.01 \mathrm{~s}$. To maximise useful capture space, the back of the boat (stern) was not captured. No calibration was necessary as the variables of interest were temporal and not spatial.

Video analysis was conducted using open source freeware Kinovea (Version 0.8.15). In sprint kayaking, a complete stroke cycle beginning and ending on one side (e.g. from the right catch to the next right catch) is commonly considered as two strokes (Szanto, 2010). A model based on the contact area of the paddle blade relative to the water was used to identify four meaningful positions of the stroke (McDonnell et al., 2012). In the model by McDonnell et al. (2012), the four positions (catch, immersion, extraction and release) separate the stroke phases (entry, pull, exit and aerial), where the entry, pull and exit are collectively the water phase (Photo 2). The catch occurred at the first contact between the paddle blade and water, immersion was when the blade was first fully submerged, extraction was the last instance of full blade submersion, and release was the last contact between the blade and water. From here on, the term "release" refers to the position of last contact between the blade and water, while "exit" refers to the phase between extraction and release.

To quantify stroke synchronisation within a $\mathrm{K} 2 \mathrm{crew}$, an offset variable was calculated as the timing difference in milliseconds (ms) of the back paddler compared to the front paddler, where offset (milliseconds) = Timing of the back paddler - Timing of the front paddler. Beginning from the $4^{\text {th }}$ right stroke, the offset was measured at four positions (catch, immersion, extraction and release) for every right stroke of both paddlers. Based on pilot work, the first three right-side strokes were excluded in order for the analysis to focus on stroke synchronisation when the boat was near race-pace velocity. With reference to the front paddler, the offset of the back paddler could be negative, zero or positive. Photo $3 a$ (top) illustrates an example of negative offset, where the back paddler catches before the front paddler. Conversely, Photo $3 \mathrm{~b}$ (bottom) shows an example of positive offset where the front paddler catches before the back paddler. The offset is zero, i.e. perfect synchronisation, if both paddlers reached the same position at the same time.

\section{Statistical Analysis}

Descriptive statistics and data visualisation were performed in Excel ${ }^{\circledR} 2016$ (Microsoft Corporation, Redmond, WA, USA). To illustrate stroke synchronisation profiles, strokeby-stroke offsets at each position were plotted in radar charts. An annotated example of a radar chart showing offsets at the catch is shown in Figure 1. In the radar chart, data points within the shaded centre represent negative offset, where the back paddler was earlier to reach the stroke position. The white area represents positive offset, where the back paddler was later to reach the stroke position. Each concentric ring was a 50-ms increment.

To assess intra-rater reliability, one 200-m trial of a men's K2 crew comprising 40 strokes was analysed twice by the same rater (Rater 1 ) with an interval of 5 days apart. To compare interrater reliability, this selected trial was also analysed independently by Raters 2 and 3. All three raters had previous undertaken training in biomechanical video analysis and conducted independent projects. In addition, Raters 1 and 2 were former national team paddlers with ten years of competitive experience, while Rater 3 was a novice paddler with two years' experience. Both inter-rater and intra-rater reliabilities were evaluated using intra-class correlation coefficients (ICC) and standard errors of measurement (SEM) in IBM SPSS Statistics for Windows Version 23.0 (Armonk, NY). Based on guidelines (Altman, 1991), the ICCs were interpreted as slight $(<.20)$, fair (.21-.40), moderate (.41-.60), substantial (.61$.80)$ and almost perfect $(>.80)$.

\section{Results}

Table 2 summarises the intra- and interrater reliabilities statistics. Intra-rater reliability of Rater 1 was almost perfect, where the ICC ranged from .87 to 1.00 , and SEM from 0 to $5 \mathrm{~ms}$. Interrater reliability was substantial to almost perfect, where the ICC ranged from .72 to .94, and SEM from 2 to $6 \mathrm{~ms}$. All SEM values were less than 8 $\mathrm{ms}$, which was the time taken for one frame of the $120 \mathrm{~Hz}$ captured videos. In the cases where interrater reliability was substantial (i.e. ICC between .61 - .80), further analyses were conducted to examine the reliability between Raters 1 and 2 (both experienced paddlers) and between Raters 1 (experienced) and 3 (novice). At the immersion 
position, inter-rater reliability was higher for Raters 1 and 2 (ICC .89 [.80, .94]) than Raters 1 and 3 (ICC .73 [.55, .85]). At the extraction, inter-rater reliability was also higher for Raters 1 and 2 (ICC $.87[.78, .93]$ ) than Raters 1 and 3 (ICC .72 [.53, $.84])$.

Table 3 shows the 200-m time trial performance and stroke synchronisation characteristics for each of the six crews. The order within each crew indicates the seating arrangement. For example, in M1/M2, M1 was the front paddler and M2 was the back paddler. The magnitude and direction of mean absolute offset varied considerably among the six crews, and also across the four stroke positions. On average, 35 strokes were analysed and the mean offset was 17 $\mathrm{ms}$, or $5.7 \%$ of water phase duration. All crews were more synchronised at the catch (11 ms, 3.8\%) than the release $(21 \mathrm{~ms}, 7.2 \%)$, but were not consistent for the immersion and extraction positions.
Stroke-by-stroke offsets at each position for each crew were illustrated in radar charts (Figure 2). Four different stroke synchronisation profiles were identified from comparing the back paddler to the front paddler. Profiles A and B characterised differences in water phase duration between the front and back paddlers. For the fastest men's crew of M1/M2, the back paddler had longer water phase duration by catching earlier and releasing later (Profile A). In contrast, the back paddler in the fastest women's crew of W1/W2 had shorter water phase duration by catching later, but releasing earlier (Profile B). Profiles C and D identified whether the back paddler was consistently lagging or leading the front paddler. For crews M3/M4 and M5/M6, there was a positive offset where the back paddler was lagging (Profile C). In contrast, the back paddlers of crews W3/W4 and W5/W6 were leading their front paddlers (Profile D).

\section{Table 1}

Participant characteristics and individual K1 200-m performance of 12 national team paddlers ( 6 males and 6 females).

\begin{tabular}{lccccccccccc}
\hline Men & $\begin{array}{c}200- \\
\mathrm{m} \\
(\mathrm{s})\end{array}$ & $\begin{array}{c}\text { Heig } \\
\mathrm{ht} \\
(\mathrm{m})\end{array}$ & $\begin{array}{c}\text { Mass } \\
(\mathrm{kg})\end{array}$ & $\begin{array}{c}\text { Age } \\
(\mathrm{y})\end{array}$ & $\begin{array}{c}\text { Exp } \\
(\mathrm{y})\end{array}$ & $\begin{array}{c}\text { Wom } \\
\text { en }\end{array}$ & $\begin{array}{c}200- \\
\mathrm{m} \\
(\mathrm{s})\end{array}$ & $\begin{array}{c}\text { Heig } \\
\text { ht } \\
(\mathrm{m})\end{array}$ & $\begin{array}{c}\text { Mass } \\
(\mathrm{kg})\end{array}$ & $\begin{array}{c}\text { Age } \\
(\mathrm{y})\end{array}$ & $\begin{array}{c}\text { Exp } \\
(\mathrm{y})\end{array}$ \\
\hline M1 & 36.8 & 1.68 & 76.0 & 25 & 12 & W1 & 42.0 & 1.63 & 60.3 & 24 & 7 \\
M2 & 34.9 & 1.71 & 76.9 & 23 & 10 & W2 & 44.5 & 1.60 & 49.2 & 25 & 8 \\
M3 & 37.8 & 1.76 & 72.1 & 30 & 13 & W3 & 43.5 & 1.54 & 56.2 & 27 & 10 \\
M4 & 36.8 & 1.73 & 70.1 & 21 & 8 & W4 & 43.1 & 1.65 & 58.3 & 22 & 7 \\
M5 & 36.8 & 1.64 & 67.4 & 27 & 14 & W5 & 43.1 & 1.66 & 56.6 & 32 & 15 \\
M6 & 39.4 & 1.73 & 69.5 & 26 & 13 & W6 & 45.0 & 1.59 & 50.8 & 22 & 5 \\
\hline Mea & 37.0 & 1.71 & 72.0 & 25 & 11.7 & Mean & 44.0 & 1.61 & 55.2 & 25 & 8.7 \\
n & & & & & & & & & & &
\end{tabular}

Note. Exp $=$ competitive paddling experience. Each paddler was identified by a participant number (e.g. M1). 
Table 2

Intra- and inter-rater reliabilities of manual video analysis for stroke synchronisation in sprint kayak $\mathrm{K} 2$ crews.

\begin{tabular}{lcccccc}
\hline & \multicolumn{3}{c}{ Intra-rater } & \multicolumn{3}{c}{ Inter-rater } \\
\hline & ICC & $95 \%$ CI & $\begin{array}{r}\text { SEM } \\
(\mathrm{ms})\end{array}$ & ICC & $95 \%$ CI & $\begin{array}{c}\text { SEM } \\
(\mathrm{ms})\end{array}$ \\
\hline Catch & .97 & $.95, .99$ & 2 & .85 & $.77, .91$ & 5 \\
Immersion & .87 & $.76, .93$ & 5 & .74 & $.61, .84$ & 6 \\
Extraction & .93 & $.85, .96$ & 3 & .72 & $.58, .83$ & 6 \\
Release & 1.00 & $1.00,1.00$ & 0 & .94 & $.90, .96$ & 2 \\
\hline
\end{tabular}

Note. ICC = intra-class correlation, $C I=$ confidence interval (lower bound, upper bound), SEM = standard error of measurement.

Table 3

Descriptive statistics of 200-m time trial performance and offset characteristics for each of the six $K 2$ crews.

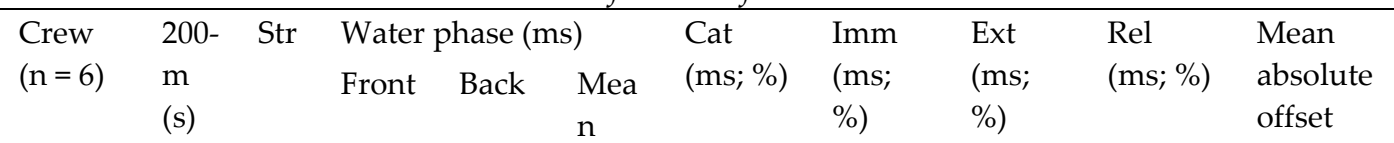

(\%)

\begin{tabular}{lllllllllll}
\hline M1/M2 & 35.0 & 36 & 253 & 281 & 267 & $-6 ; 2.2$ & $-9 ; 3.4$ & $30 ;$ & $22 ; 8.2$ & 6.3 \\
M3/M4 & 35.9 & 36 & 256 & 260 & 258 & $16 ; 6.2$ & $0 ; 0.0$ & $32 ;$ & $20 ; 7.8$ & 6.6 \\
& & & & & & & & 12.4 & & \\
M5/M6 & 38.5 & 34 & 279 & 281 & 280 & $11 ; 3.9$ & $7 ; 2.5$ & $14 ; 5.0$ & $13 ; 4.6$ & 4.0 \\
W1/W2 & 41.7 & 36 & 327 & 281 & 304 & $11 ; 3.6$ & $22 ; 7.2$ & $-53 ;$ & $-35 ;$ & 10.0 \\
& & & & & & & & 17.4 & 11.5 & \\
W3/W4 & 44.0 & 37 & 300 & 300 & 300 & $-8 ; 2.7$ & $-2 ; 0.7$ & $-4 ; 1.3$ & $-9 ; 3.0$ & 1.9 \\
W5/W6 & 46.7 & 33 & 341 & 328 & 335 & $-13 ; 3.9$ & $-16 ; 4.8$ & $-17 ; 5.1$ & $-26 ; 7.8$ & 5.4 \\
\hline Mean & & 35 & & & 313 & $11 ; 3.8$ & $9 ; 3.1$ & $25 ; 8.7$ & $21 ; 7.2$ & 5.7
\end{tabular}

Note. Str $=$ number of strokes analysed, $m s=$ milliseconds, Cat $=$ catch,

$I m m=$ immersion, $E x t=$ extraction, $R e l=$ release. The order within each crew indicates

the seating arrangement. For example, in a K2 crew of M1/M2, M1 was the front paddler and M2 was the back paddler. The number of strokes analysed began from the $4^{\text {th }}$ right-side stroke. 


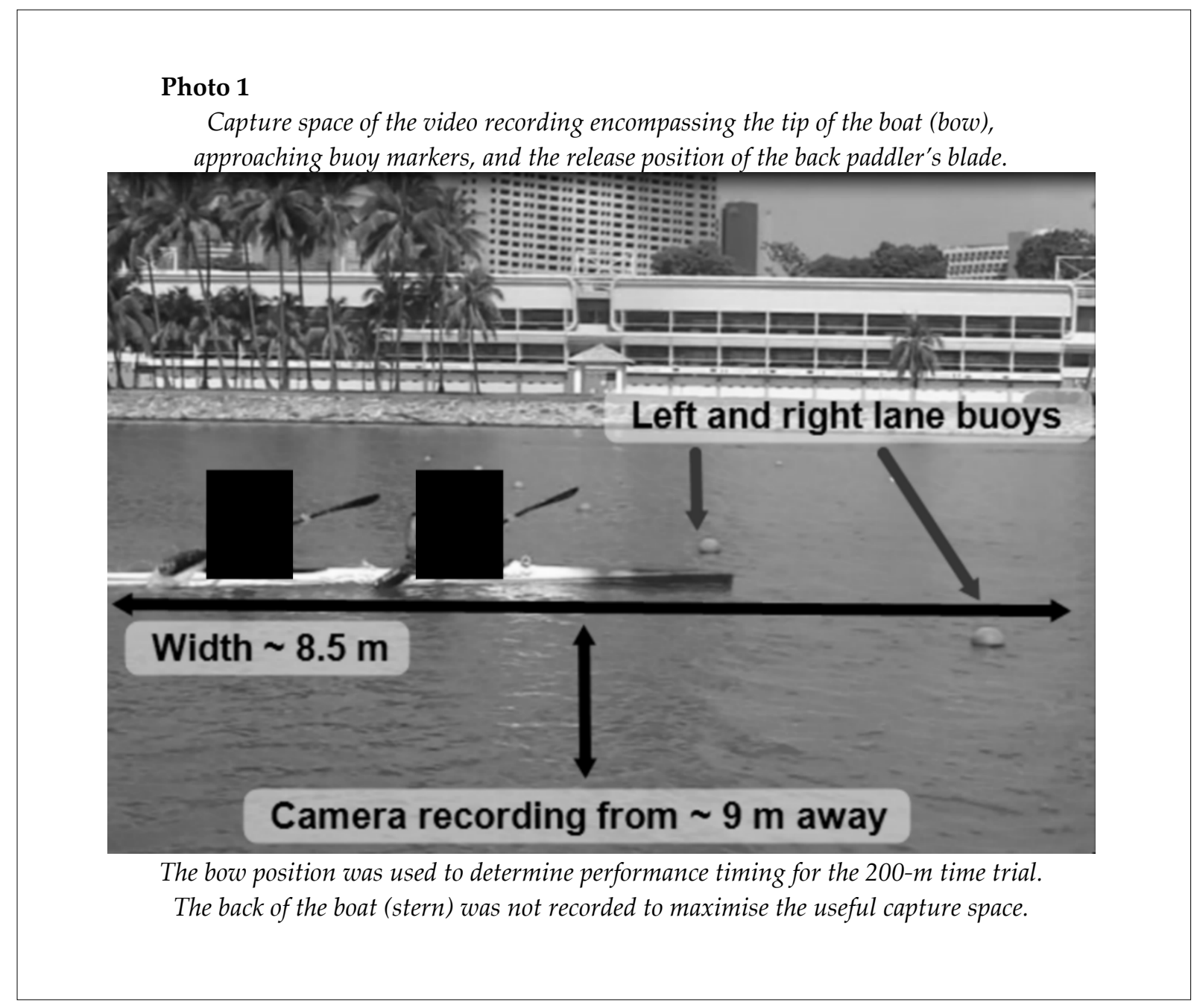

Photo 2

Phases of the kayaking stroke (entry, pull, exit and aerial) separated

by phase-defining positions (catch, immersion, extraction and release).

Stroke phases

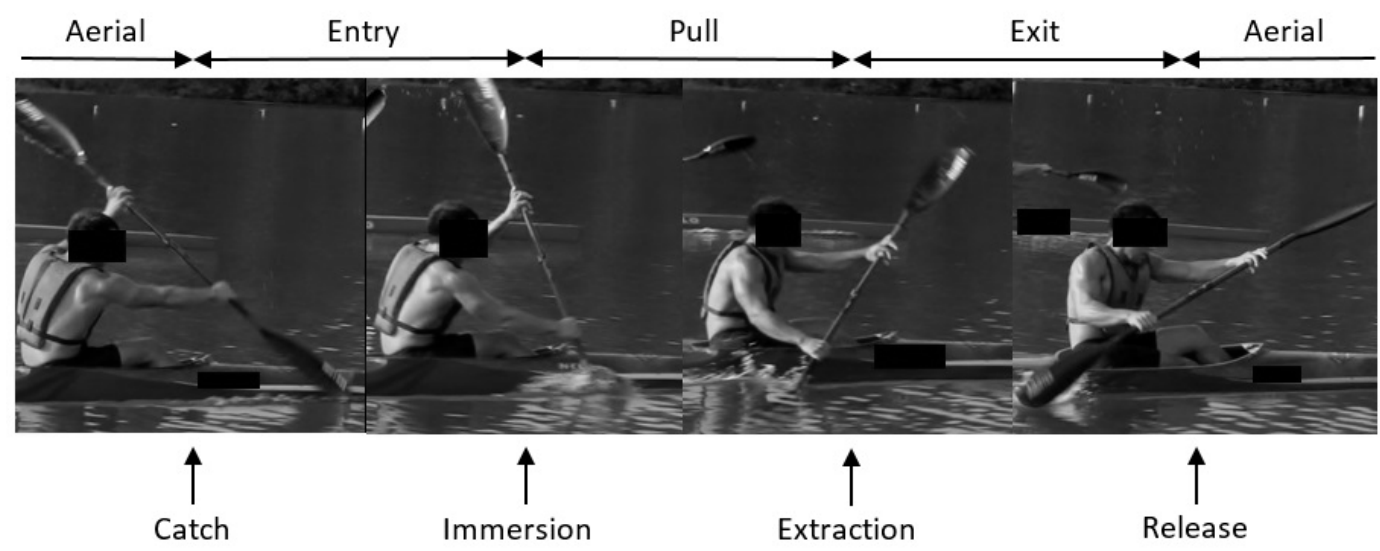

Phase-defining positions 
Photo 3

Examples of offset types for a sprint kayak K2 crew at the catch position.

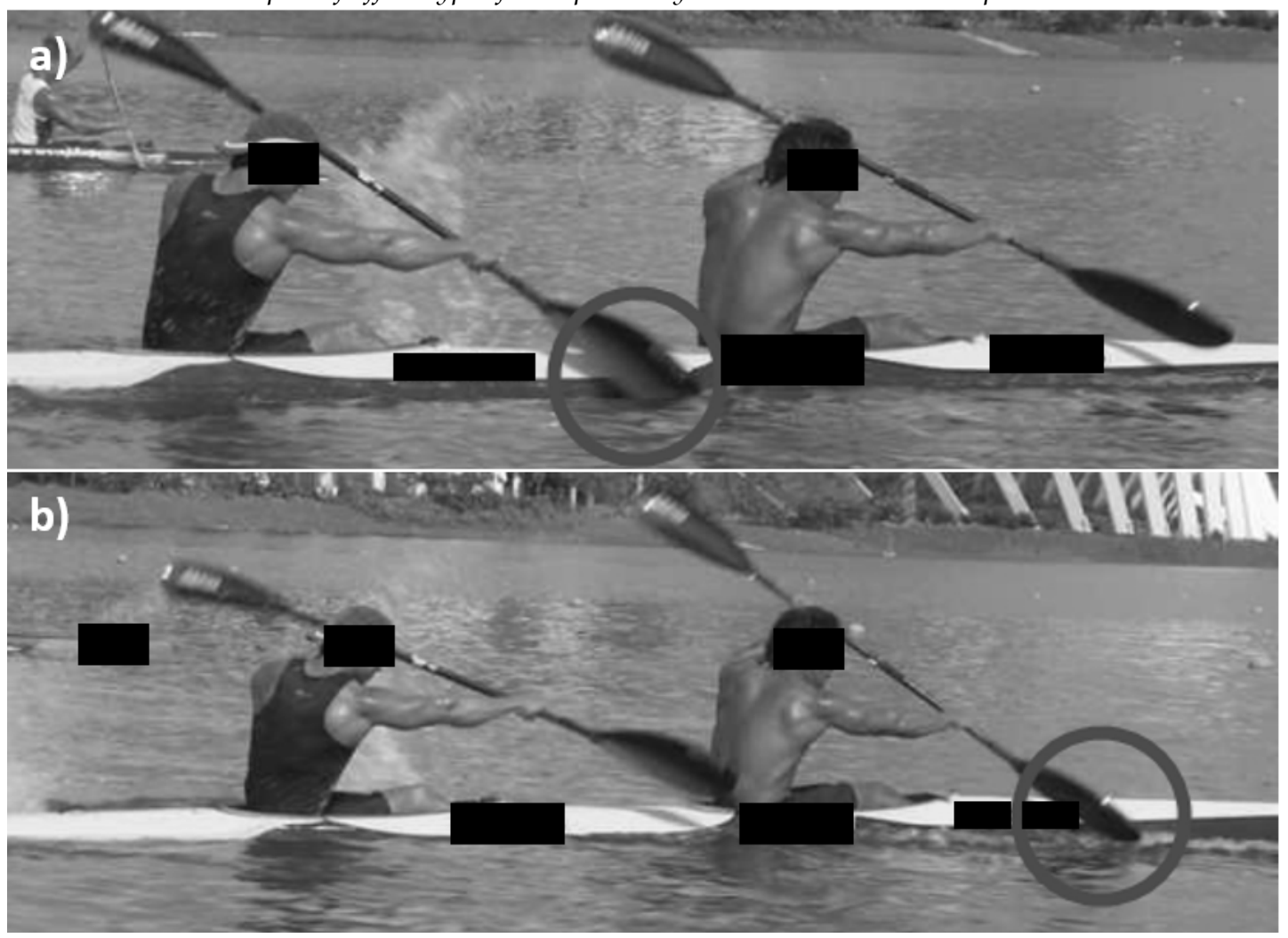

(a) A negative offset is where the back paddler's blade catches before the front paddler's.

(b) A positive offset is where the front paddler's blade catches before the back paddler's.

A zero offset (not shown) is where both paddlers catch at the same time. 


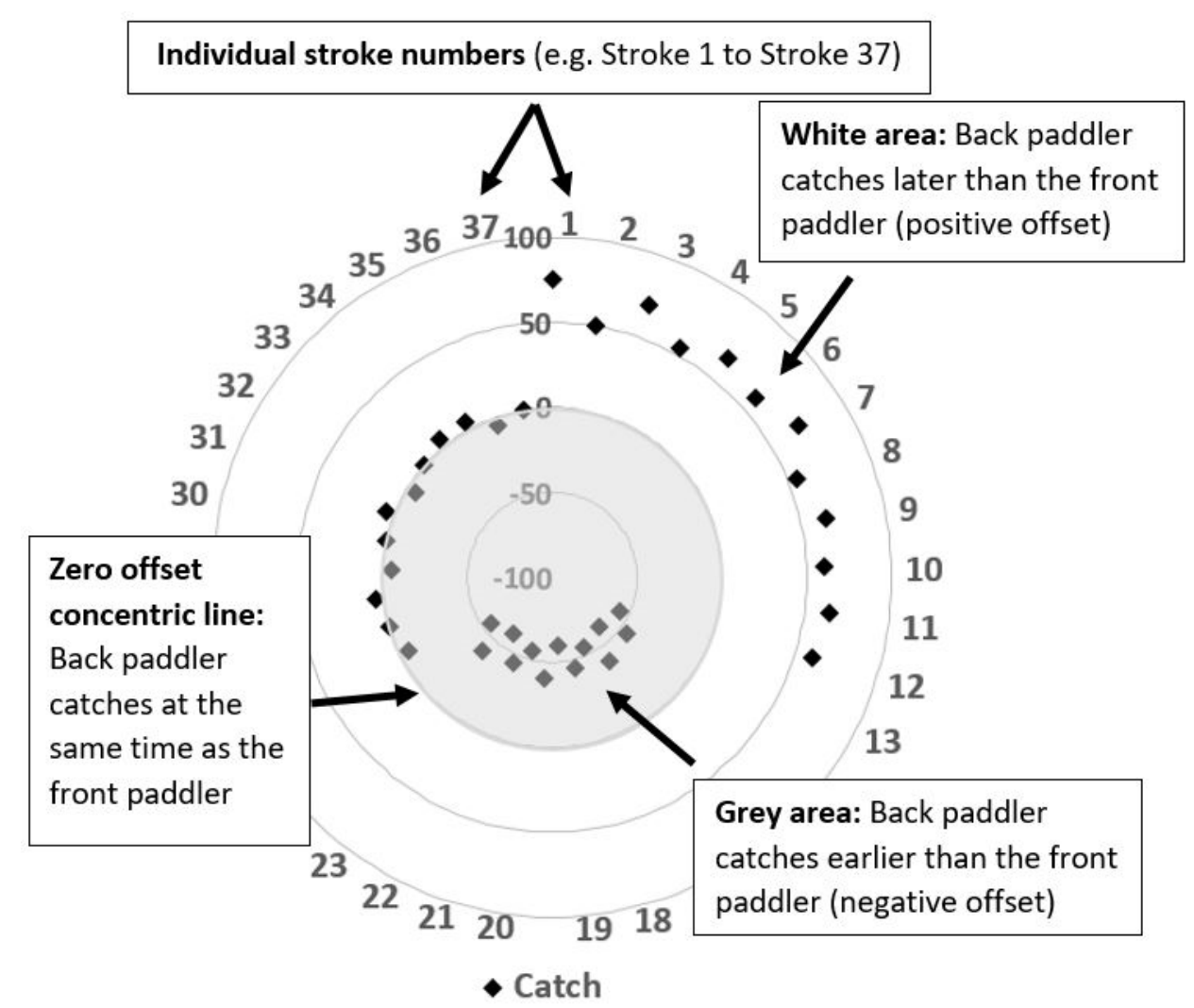

Figure 1

Annotated radar chart illustrating stroke-by-stroke offset at each position. The numbers at the rim (1 to 37) are the consecutive right-side strokes. Each concentric ring is a 50-milliseconds increment. 


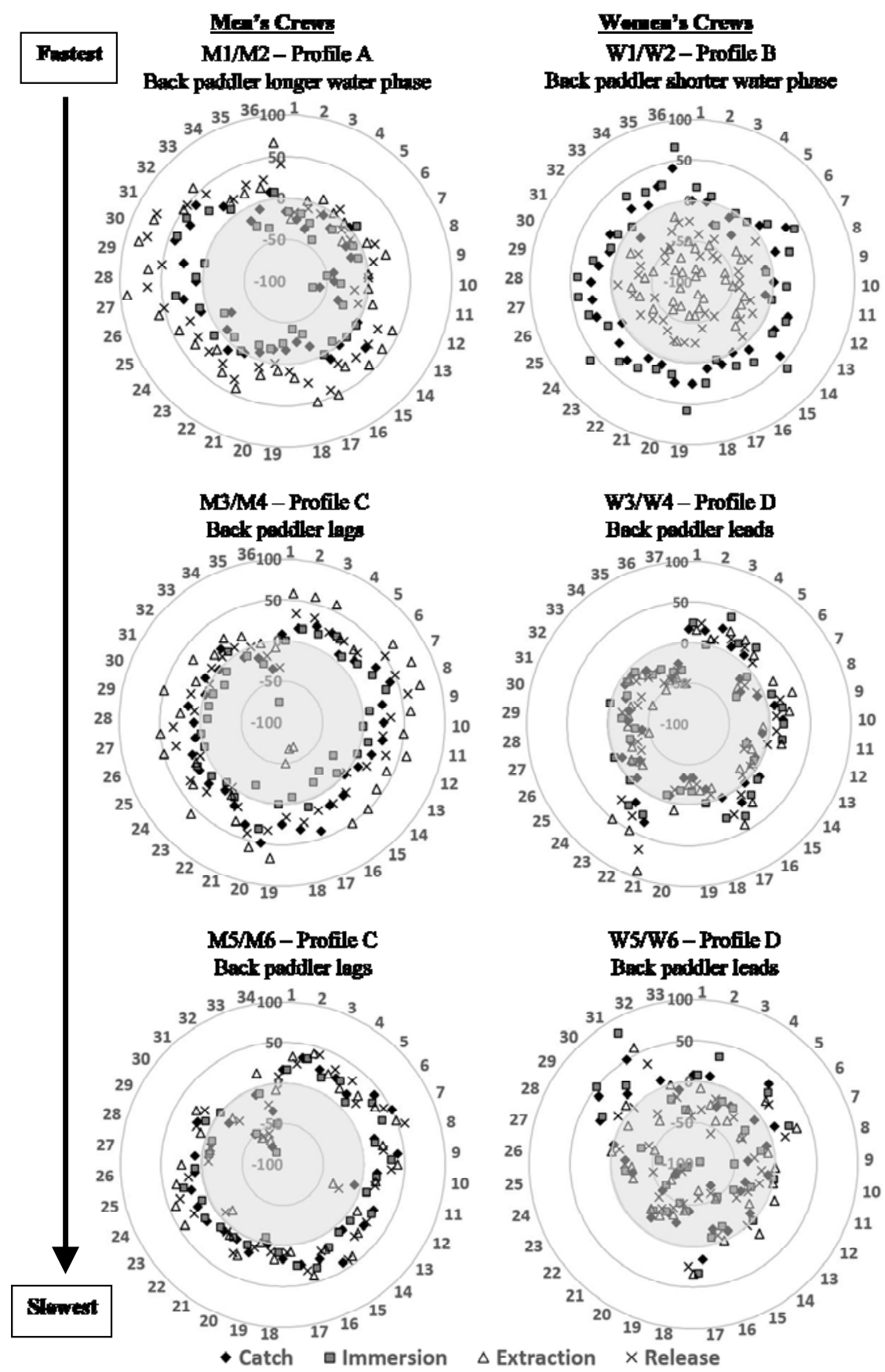

Figure 2

Radar charts illustrating stroke-by-stroke offset at each position for each K2 crew.

Four different stroke profiles were identified from comparing the back paddler to the front paddler: A. Back paddler longer water phase,

$B$. Back paddler shorter water phase,

C. Back paddler lags, D. Back paddler leads. 


\section{Discussion}

The aims of this study were to quantify stroke synchronisation in crew boat sprint kayaking using a video-based method, and to assess the intra- and inter-rater reliabilities of this method. A four-position stroke model (McDonnell et al., 2012) was adopted to identify the timing difference (offset) between two paddlers in a K2 crew. As hypothesised, the proposed method was reliable in quantifying stroke synchronisation of sub-elite sprint kayak $\mathrm{K} 2$ crews.

The reliability analysis results are promising, as intra-rater reliability was almost perfect, while inter-rater reliability ranged from substantial to almost perfect (Table 2). Given that the proposed method did not require expensive equipment or complex data collection or analysis procedures (e.g. no calibration required, free software), this method is practical for coaches and sport scientists working with sprint kayakers. Our findings also showed better reliability with raters who have more years of competitive paddling experience, but it is cautioned that the analyses were based on only two experienced and one less experienced raters. Furthermore, the intra-rater reliability was evaluated for one trial only, hence the reliability may be affected by different lighting and wave conditions.

The video-based method identified a variety of stroke synchronisation profiles across the sample of six K2 crews from a national team. As a percentage of the water phase duration, the mean offset ranged from 1.9 to $10.0 \%$. To the best of our knowledge, this is the first study to quantify the magnitude of stroke synchronisation in crew boat sprint kayaking. While there are no data for direct comparison in sprint kayaking, our findings mirror a study on crew coordination of elite rowing pairs which found that crews were more synchronised at the catch compared to the release (Hill, 2002). Interestingly, the mean offset values of $11 \mathrm{~ms}$ (catch) and $21 \mathrm{~ms}$ (release) in our study were almost identical to those of the rowing crews (11 ms at the catch and $22 \mathrm{~ms}$ at the release). Hill (2002) suggested that crew members were better able to perceive a higher force at the catch compared to the small force at the release, thus the catch was an important trigger for crew boat synchronisation.

The four stroke synchronisation profiles were identified from comparing the back paddler to the front paddler: A. Back paddler had longer water phase duration, B. Back paddler had shorter water phase duration, C. Back paddler was lagging, D. Back paddler was leading. The fastest male and female crews used directly contrasting profiles (Profile A versus Profile B). Profile A is where the back paddler had longer water phase duration than the front paddler by catching first and releasing later. This profile is in agreement with photographic observations of some of the top international level sprint kayak crews (Tellez et al., 2015). In contrast, the fastest female crew of W1/W2 displayed Profile B, where the back paddler had shorter water phase duration by catching later and releasing first. Based on the individual performance of these participants from their K1 timings (Table 1), back paddler M2 was faster than front paddler M1, and front paddler W1 was faster than back paddler W2. These two cases showed a tendency for the faster K1 paddler to take a longer stroke when placed in a K2 crew boat. Based on the methods established in the present study, future studies may further investigate the extent to which stroke synchronisation might affect performance in crew boat sprint kayaking.

In elite rowing where the research is more established, Hill (2002) showed that a crew could be more synchronous in timing (3.1\% difference), but concurrently, less synchronised in their force profiles $(7.7 \%$ difference). Currently, force measurements have been made for paddlers in a single kayak (Gomes et al., 2015; Macdermid and Fink, 2017), but not yet in a crew boat. However, the commercially available instrumented paddles are costly and may not be accessible to many coaches, athletes, and sports scientists. There are also occasions (e.g. competitions) when athletes prefer to use their own paddles rather than instrumented paddles. Under these circumstances, video-based analysis as reported in the present study can be a practical tool to obtain reliable and useful information on stroke synchronisation in a crew boat.

There were several limitations to this study. First, the study design was delimited to testing at the 200-m distance and only the $\mathrm{K} 2 \mathrm{crew}$ boats to serve as a base level for future comparisons. The $200-\mathrm{m}$ is the shortest distance for sprint kayaking, while the $\mathrm{K} 2$ is the most basic unit of a crew boat. Future research may consider 
the inclusion of the bigger $\mathrm{K} 4$ crew boat as well as multiple race distances, i.e. 500-m and 1000-m. Second, this study was limited by the small sample size of six crews from a national team. It is possible that synchronisation offsets and profiles may differ depending on the proficiency level. For example, novice paddlers may be less synchronised in crew boats compared to welltrained or elite paddlers. Finally, the video analysis method was performed by raters with competitive paddling experience; the intra- and inter-rater reliabilities are not known for raters without any paddling experience.
In conclusion, this study introduced a video-based method to quantify stroke synchronisation in crew boat sprint kayaking. Intra- and inter-rater reliabilities of this method was almost perfect for raters with substantial competitive experience in sprint kayaking. Crews were more synchronised at the catch compared to the release positions of the stroke. However, the synchronisation profiles of the crews varied considerably. For example, the best performing male and female crews had directly contrasting profiles, which were also different from the other four crews.

\section{Acknowledgements}

We would like to thank Coach Balazs Babella and the Singapore National Kayak Sprint team for participating in the study. We also acknowledge support from the Nanyang President's Graduate Scholarship, Nanyang Technological University. No conflict of interest was reported by the authors.

\section{References}

Altman DG. Practical statistics for medical research. Padstow, London: Chapman \& Hall, 398-403; 1991

Borges TO, Bullock N, Coutts AJ. Pacing characteristics of international sprint kayak athletes. Int J Perform Anal Sport, 2013; 13: 353-364

de Brouwer AJ, de Poel HJ, Hofmijster MJ. Don't rock the boat: how antiphase crew coordination affects rowing. PLoS One, 2013; 8: e54996

Gomes BB, Ramos NV, Conceição FA, Sanders RH, Vaz MA, Vilas-Boa JP. Paddling force profiles at different stroke rates in elite sprint kayaking. J Appl Biomech, 2015; 31: 258-263

Hill H. Dynamics of coordination within elite rowing crews: evidence from force pattern analysis. J Sport Sci, 2002; 20: 101-117

Jackson PS. Performance prediction for Olympic kayaks. J Sport Sci, 1995; 13: 239-245

King A, de Rond M. Boat race: rhythm and the possibility of collective performance. Brit J Sociol, 2011; 62: 565-585

Martin TP, Bernfield JS. Effect of stroke rate on velocity of a rowing shell. Med Sci Sport Exer, 1980; 12: 250256

Macdermid PW, Fink, PW. The validation of a paddle power meter for slalom kayaking. Sport Med Int Open, 2017; 1: E50-E57

McDonnell LK, Hume PA, Nolte V. An observational model for biomechanical assessment of sprint kayaking technique. Sport Biomech, 2012; 11: 507-523

Michael JS, Smith R, Rooney KB. Determinants of kayak paddling performance. Sport Biomech, 2009; 8: 167179

Robinson MG, Holt LE, Pelham TW, Furneaux K. Accelerometry measurements of sprint kayaks: the coaches' new tool. Int J Coaching Sci, 2011; 5: 45-56 
Szanto C. ICF canoe sprint coaching manual level 2 and 3. Lausanne: International Canoe Federation, 79-84; 2010

Tellez JC, Hernandez JLS, Imaz X. Training sprint canoeing. Spain: Real Federacion Espanola de Pirguismo, 227-251; 2015

Wing AM, Woodburn C. The coordination and consistency of rowers in a racing eight. J Sport Sci, 1995; 13: 187-197

\section{Corresponding author:}

\section{Pui Wah Kong}

Physical Education and Sports Science Academic Group, National Institute of Education, Nanyang Technological University, Singapore

1 Nanyang Walk, Singapore, 637616

Phone: (65) 62196213

Fax: (65) 6896-9260

puiwah.kong@nie.edu.sg 